\title{
MANIA Complex - Hardware and Software for the Investigation of Optical Variability on Time Scales $10^{-7}-10^{2} \mathrm{~s}$
}

\author{
G.M. Beskin, S.I. Neizvestny, V.L. Plokhotnichenko, A.I. Zhuravkov \\ Special Astrophysical Observatory, Nizhnij Arkhyz, Karachaevo-Circassia, 357147, \\ Russia
}

One of the basic astrophysical problems is the search for and investigation of brightness fluctuations of celestial objects on time scales comparable with the mean time between registered photons. To facilitate this a special mathematical formalism was developed (Shwartsman 1977, Plokhotnichenko 1983).

At the Special Astrophysical Observatory, photometric equipment has been developed which allows these ideas to be explored observationally and which also allows to study light curves of astrophysical objects using classical methods.

This equipment and these methods are used in the search for and study of variable objects with time resolution $10^{-7} \mathrm{~s}$ in the MANIA (Multichannel Analysis of Nanosecond Intensity Alterations) experiment. The hardware consists of a photon-counting photometer for synchronous detection in different colour bands, a specialized "time-code" converter (Quantochron 3-16), measuring photon arrival times with an accuracy of $20 \mathrm{~ns}$ and a PC AT 386 and DAT-cassette recorder. Our equipment allows the uninterrupted accumulation of $10^{8}$ photocounts with a rate up to $375 \mathrm{kHz}$ without distortions in $2^{8}$ or $2^{16}$ parallel channels (Zhuravkov et al. 1994). The software permits the investigation of variability on time scales $10^{-7}-10^{2} \mathrm{~s}$.

Two different versions of this complex are installed on the 6-m telescope of the Special Astrophysical Observatory and on the 2.15-m telescope of the Leoncito Observatory in Argentina.

The following astrophysical results have been obtained recently:

- Observations of 40 objects - candidates for isolated black holes - have shown that their luminosity is constant on time scales of $10^{-6}-10^{2} \mathrm{~s}$. On the basis of these results we obtained the upper limit of the number of black holes $5 \cdot 10^{-4}$ of the number of usual stars (Swartsman at al. 1989).

- A light curve of the Crab pulsar with a resolution of $3.3 \mu$ s was obtained (see Fig. 1). The main pulse width at $95 \%$ of maximum level is $\sim 200 \mu \mathrm{s}$. There are no modulations which exceed $10 \%$ on $5 \mu$ s. (Swartsman at al. 1988a).

- The pulsar PSR $1937+21(P=1.6 \mathrm{~ms})$ has a pulsating component fainter than $B=26^{\mathrm{m}} 2$. As a consequence, the lower limit of the distance to the pulsar was determined to be $5 \mathrm{kpc}$ (Swartsman at al. 1988b).

- The temporal structure of 100 flashes of UV Cet type stars was investigated. On the basis of these data it was proven that the thermal hydrodynamic mechanism takes place in these objects (Swartsman et al. 1988c). 


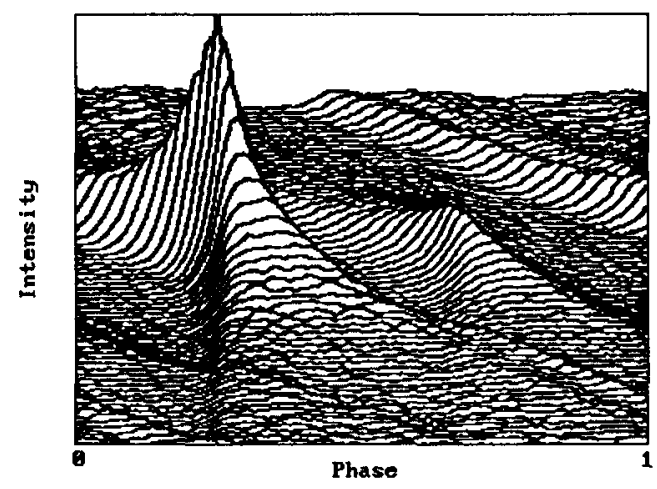

Fig. 1. Series of Crab pulsar light curves with close periods

- Non-thermal flashes were detected in observations of V616 Mon = A062000 and MXB 1735-44. Their duration is between $1 \mathrm{~ms}$ and $0.2 \mathrm{~s}$, their rise time between $0.1 \mathrm{~ms}$ and $0.1 \mathrm{~s}$. Fine structure on time scales of $5-10 \mathrm{~ms}$ was detected. Brightness temperatures exceed $10^{8}-10^{9} \mathrm{~K}$ (Swartsman at al. 1989, Beskin et al. 1994).

- The variability of V518 Per = GRO J0422+32=X-ray nova Per 1992 in the high optical state $(V<15 \mathrm{~m})$ on time scales from $4 \mathrm{~ms}$ to $200 \mathrm{~s}$ has been detected (Bartolini et al. 1994). The shortest flashes have nonthermal origin since their brightness temperatures exceed $10^{8} \mathrm{~K}$.

\section{References}

Bartolini C, Guarnieri A., Piccioni A., Beskin G., Neizvestny S., 1994, ApJS 92, 455

Beskin G., Neizvestny S., Mitronova S., Plokhotnichenko V., Popova M., Benvenuto O., Feinstein C., Mendez M., 1994, A\&A 289, 141

Plokhotnichenko V.L., 1983, Soobshch. Spets. Astrofiz. Obs. 38, 28

Shvartsman V.F., 1971, Astron. Zh. 48, 479 = Sov. Astron. J. 15, 377

Shvartsman V.F., Beskin G.M., Plokhotnichenko V.L., 1988a, in The Physics of Neutron Stars. Pulsars and Bursters, Leningrad, p. 178

Shvartsman V.F., Beskin G.M., Plokhotnichenko V.L., 1988b, in The Physics of Neutron Stars. Pulsars and Bursters, Leningrad, p. 184

Shvartsman V.F., Beskin G.M., Gershberg R.E., Plokhotnichenko V.L., Pustilnik, L.A., 1988c, Pis'ma v Astron. Zh. 14, 223

Shvartsman V.F., Beskin G.M., Mitronova S.N., 1989, Pis'ma v Astron. Zh. 15, 337

Zhuravkov A.V., Pimonov A.A., Plokhotnichenko V.L., 1994, Bull. Spec. Astrophys. Obs. 37,159 